\title{
1,4-Dimethylenespiropentane: A Unique Model System for Studying Fermi Resonance in Raman Optical Activity
}

\author{
Werner Hug, ${ }^{[a]}$ Jacques Haesler, ${ }^{[a]}$ Sergei I. Kozhushkov, ${ }^{[b]}$ and \\ Armin de Meijere ${ }^{[b]}$
}

The $C_{2}$-symmetric title compound, a small, rigid hydrocarbon molecule with two distinct, strongly interacting vibrational chromophores, represents a unique model system for testing computational approaches to Raman optical activity (ROA) beyond the isolated molecule and the harmonic approximation. We show that the experimental Raman and ROA spectra are marked by the presence of strong Fermi resonances, and that the shape and relative size of the bands which we attribute to such resonances strongly depend on the solvent environment of the molecule. On the other hand, the dominant computed ROA couplet, arising from the $C=C$ stretching vibrations, is absent in the spectra measured in the condensed phase. The strong dependence of the computed size of the couplet on the description of the diffuse part of the electron distribution suggests that this absence is due to nonspecific interactions in the condensed phase.

\section{Introduction}

Vibrational optical activity is a powerful spectroscopic tool for studying the structure of molecules which do not possess improper rotation symmetry elements. Moreover, the ab initio computation of Raman optical activity (ROA) and of vibrational circular dichroism (VCD) has proved highly successful, ${ }^{[1-5]}$ far more so than that of electronic circular dichroism. This is remarkable, as with few exceptions ${ }^{[6,7]}$ the data computed for free molecules have been compared with those measured in the liquid phase. The use of the harmonic approximation has likewise been found to yield satisfactory computed spectra, except for the expected difference between computed and observed vibrational frequencies, and except for the occasional band in experimental spectra due to an overtone or combination frequency in Fermi resonance with a fundamental, a feature which inherently cannot be accounted for without taking anharmonicity into account. For small molecules, and in the absence of specific interactions in the condensed phase, agreement between computed and measured Raman and ROA spectra can actually be astounding. ${ }^{[1]}$

1,4-Dimethylenespiropentane ${ }^{[8]}$ looks, at the outset, like another model compound well-suited for confirming the reliability of the ab initio computation of vibrational optical activity. The $C_{2}$-symmetric rigid molecule has no conformational freedom, and strong, specific interactions in the liquid phase are not expected for a pure hydrocarbon compound. Moreover, the small size of the molecule permits a relatively high-level computational approach.

Some time ago, ${ }^{[9]}$ the ROA backscattering spectrum of

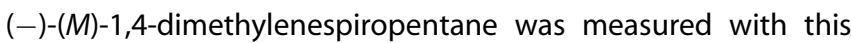
expectation on a novel instrument capable of yielding highprecision ROA data. ${ }^{[10,11]}$ The subsequent calculation of the
Raman and ROA spectra of the compound proved rather disappointing. The salient computed feature, a very large couplet centered near $1820 \mathrm{~cm}^{-1}$ and resulting from the interaction of the stretching motions of the two $\mathrm{C}=\mathrm{C}$ double bonds, is not present in the measured spectrum. Conversely, two intense and strongly polarized bands are found in the experimental Raman spectrum in the $1700-1800 \mathrm{~cm}^{-1}$ region, while only one is predicted. Likewise, in the $900-950 \mathrm{~cm}^{-1}$ range, two strong, partly separated polarized Raman bands are observed, but a polarized and a depolarized band, the latter corresponding to two close-lying vibrational states, are expected from the computed data.

Recent higher level computations ${ }^{[5]}$ have not improved on this unsatisfactory state of affairs. It was therefore decided to resynthesize the $(P)$-enantiomer of 1,4-dimethylenespiropentane so that the Raman and ROA spectra of this unique chiral molecule could be newly measured and compared with the data of the previously measured $(M)$-enantiomer. Our concerns were the purity of the originally measured samples (the molecule is not stable at room temperature), the verification of the precision of the spectroscopic measurements, and the assess-

[a] Prof. Dr. W. Hug, Dr. J. Haesler

Department of Chemistry, University of Fribourg

Ch. du Musée 9, 1700 Fribourg (Switzerland)

Fax: (+41) 26-300-97-37

E-mail:werner.hug@unifr.ch

[b] Dr. S. I. Kozhushkov, Prof. Dr. A. de Meijere Institut für Organische und Biomolekulare Chemie der Georg-August-Universität Göttingen

Tammannstrasse 2, 37077 Göttingen (Germany)

Fax: (+49) 551-39-3231 
ment of a potential influence of the condensed phase on the Raman and ROA data.

A further incentive for the renewed synthesis was that forward ROA scattering had become available on a newly constructed instrument. ${ }^{[12]}$ In contrast to ordinary Raman scattering, which is the same in the forward and the backward direction, ROA strongly differs for the two scattering geometries. It was thus hoped that the information contained in forward scattering ROA might help to clarify the origin of the discrepancy between measured and computed data.

\section{Backscattering Spectra of the Neat Liquids}

Experimental Data of the $(-)-(M)$ - and $(+)-(P)$ Enantiomers of 1,4-Dimethylenespiropentane

The backscattering spectra of (-)-(M)-1,4-dimethylenespiropentane are shown in Figure 1, and the newly measured spectra of $(+)-(P)-1,4$-dimethylenespiropentane in Figure 2. Figure 3

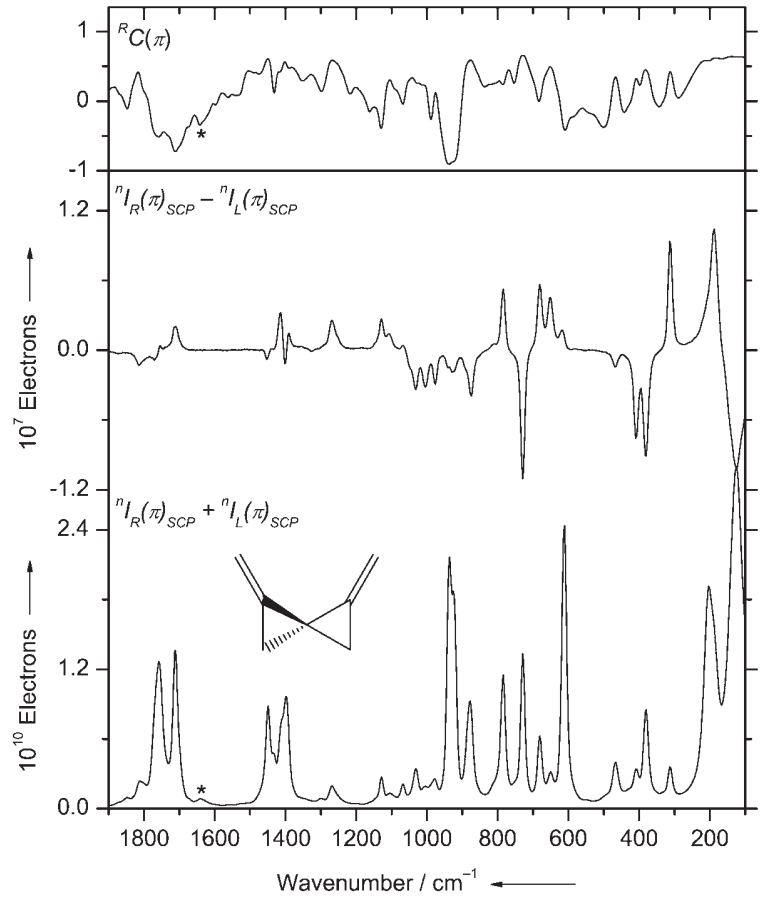

Figure 1. Experimental backscattering scattered circular polarization (SCP) Raman and ROA spectra of (-)-(M)-1,4-dimethylenespiropentane measured as neat liquid on the Zürich ROA instrument. The top curve is the degree of circularity. Exposure time $30 \mathrm{~min}$, laser power at sample $150 \mathrm{~mW}$, exciting wavelength $532 \mathrm{~nm}$, resolution $7 \mathrm{~cm}^{-1}$, average spectral width of one column of the CCD detector $2.4 \mathrm{~cm}^{-1}$, sample size $35 \mu \mathrm{L}$. The bands marked with asterisks are due to impurities (see text).

displays the superposition of the measured $\Delta$ curves of the two enantiomers, which are the ratios of the ROA and Raman intensities [Eq. (1)]:[13]

$\Delta(\pi)=\frac{{ }^{n} N_{R}(\pi)-{ }^{n} N_{L}(\pi)}{{ }^{n} N_{R}(\pi)+{ }^{n} N_{L}(\pi)}$,

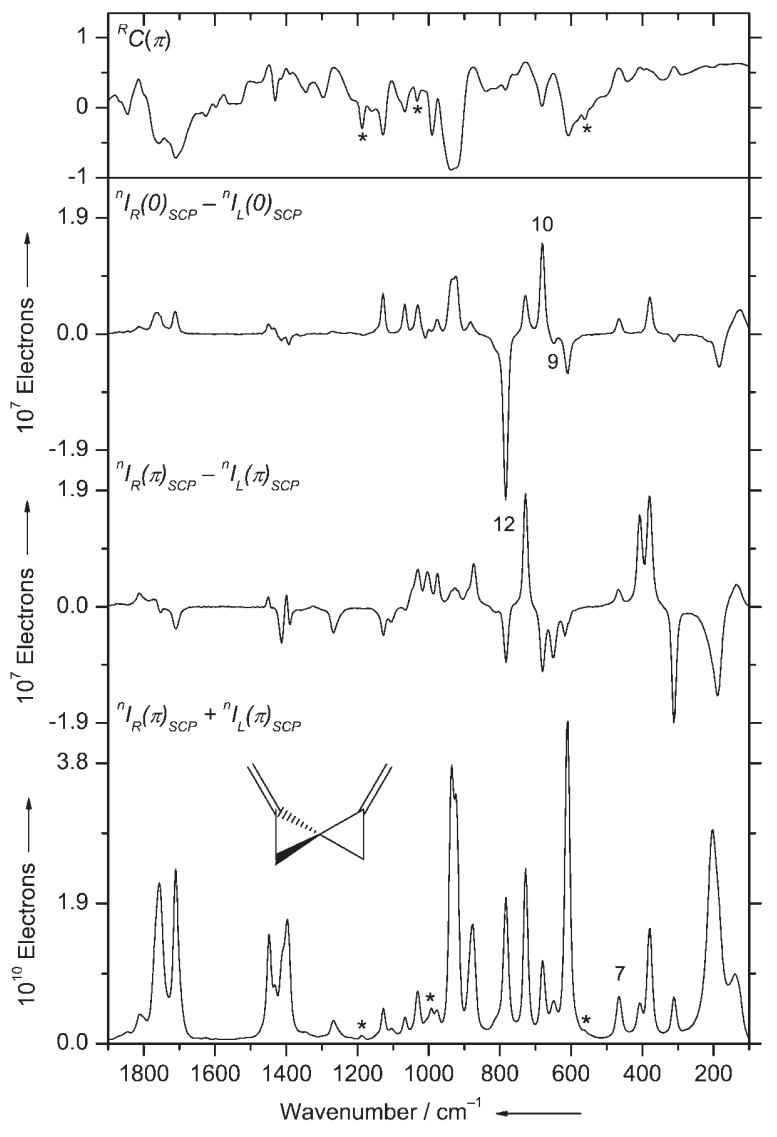

Figure 2. Experimental Raman and ROA spectra of $(+)-(P)-1,4$-dimethylenespiropentane measured as neat liquid on the Fribourg ROA instrument. From bottom to top: Raman backscattering, ROA backscattering, ROA forward scattering, degree of circularity for backscattering. Raman forward scattering is identical to backscattering except for a reduced throughput of the fiber optics used in the instrument; ROA forward scattering has therefore been adjusted to correspond to the same throughput as backscattering. Exposure time $40 \mathrm{~min}$, laser power at sample $150 \mathrm{~mW}$, exciting wavelength $532 \mathrm{~nm}$, resolution $7 \mathrm{~cm}^{-1}$, average spectral width of one column of the CCD detector $2.4 \mathrm{~cm}^{-1}$, sample size $35 \mu \mathrm{L}$. The bands marked with asterisks are due to impurities (see text).

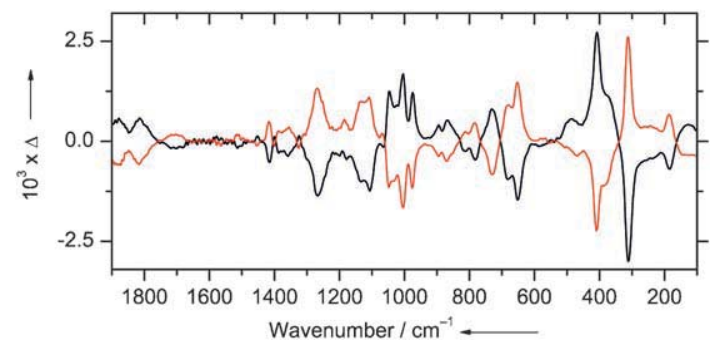

Figure 3. Comparison of the experimental $\Delta$ curves (ratio of ROA to Raman scattering) measured for backscattering on the Zürich instrument for neat $(-)-(M)$-1,4-dimethylenespiropentane (red) and on the Fribourg instrument for neat $(+)-(P)-1,4$-dimethylenespiropentane (black). Experimental parameters as in Figures 1 and 2.

where ${ }^{n} N_{R}(\pi)$ and ${ }^{n} N_{L}(\pi)$ are the numbers of the left $(L)$ and right $(R)$ circularly polarized detected photons in the backscattering $(\pi)$ direction for unpolarized $(n)$ exciting light. $\Delta$ curves 
have the virtue of being independent of experimental parameters such as the laser intensity, the measuring time, and the efficiency of the light collection optics, gratings, and detectors. Despite this advantage, experimental $\Delta$ curves are rarely published (to our knowledge, only two examples exist in the literature ${ }^{[1,3]}$, because their measurement requires the absence of instrumental offsets, a high signal-to-noise ratio, good straylight control, and clean samples devoid of fluorescence.

The curves in Figure 3, measured on two different instruments, are essentially mirror images of each other. This correspondence demonstrates the high instrumental precision of the measurements and the similar enantiomeric purity of the measured samples of the $(M)$ - and the $(P)$-enantiomers. The similarity of the two curves does not, however, exclude the presence of the same optically inactive impurity in the two samples if it were present in the same concentration. An optically active impurity, on the other hand, would show up as a deviation from the mirror symmetry of the two curves, except in the case where opposite enantiomers were present in (-)$(M)$-1,4-dimethylenespiropentane and (+)-(P)-1,4-dimethylenespiropentane in equal amounts. In order to exclude the presence of any substantial amount of impurity due to the transportation or the measurement of the fragile compounds, we re-measured the ${ }^{1} \mathrm{H}$ NMR spectrum of $(+)-(P)$-1,4-dimethylenespiropentane after it had been transported in dry ice from Göttingen to Fribourg and again after its backscattering spectra had been measured. From the combined Raman, ROA, and NMR evidence, it appears safe to conclude that sizable differences between computed and measured data are due neither to the presence of an impurity nor to instrumental errors.

This does not mean that the two samples are, except for being enantiomers, entirely equal. The degree of circularity, ${ }^{[14]}$ also included in Figures 1 and 2, can be a very sensitive probe for small differences between samples, as we have demonstrated in the case of $(-)-(M)-\sigma-[4]$ helicene. ${ }^{[1]}$ For backscattering, the degree of circularity is defined as given in Equation (2):

${ }^{R} C(\pi)=\frac{{ }^{R} N_{R}(\pi)-{ }^{R} N_{L}(\pi)}{{ }^{R} N_{R}(\pi)+{ }^{R} N_{L}(\pi)}$.

Small differences in the Raman spectra and in their degrees of circularity are indicative of small but different amounts of impurities present in the samples of $(-)-(M)-1,4$-dimethylenespiropentane and $(+)-(P)-1,4-$ dimethylenespiropentane. The bands arising from impurities are marked by asterisks in Figures 1 and 2.

From the differences in the spectra, we can conclude that the small band at $1640 \mathrm{~cm}^{-1}$ (at the low-wavenumber side of the signal of the coupled $\mathrm{C}=\mathrm{C}$ stretching vibrations discussed in detail in the following section) is due to an impurity rather than to a combination or overtone frequency, because it is only present in the sample of the $(-)-(M)$ enantiomer. The bands above $1840 \mathrm{~cm}^{-1}$, on the other hand, are almost certainly overtone and combination bands of the 1,4-dimethylenespiropentane molecule itself.

The differing appearance below about $400 \mathrm{~cm}^{-1}$ of the curves of the degrees of circularity in Figures 1 and 2 and of the $\Delta$ curves in Figure 3 is not due to impurities. The spectrum of (-)-(M)-1,4-dimethylenespiropentane was measured with the intent to get as close to the Rayleigh line as possible, so the holographic notch filter used to suppress light at the laser wavelength was tilted - the higher transmission at wavenumbers below $200 \mathrm{~cm}^{-1}$ is clearly visible in Figure 1. Depending on the properties of the notch filter, this modification can lead to a larger amount of stray light on the CCD detector of the instrument at low-wavenumber shifts, which in turn reduces $\Delta$ values and influences the measured degree of circularity. Where the Raman signal dominates, at the top of the Raman bands, the values of the degree of circularity are similar for the two samples.

\section{Comparison of Computed and Measured Data in the $\mathrm{C}=\mathrm{C}$ Stretching Region}

Figure 4 shows the computed Raman and ROA backscattering spectra of $(+)-(P)-1,4-$ dimethylenespiropentane. The ROA spectrum is dominated by a couplet due to the coupled $\mathrm{C}=\mathrm{C}$ stretching motions. The in-phase component (symmetry species $A$, vibration 30$)$ is fairly polarized $\left({ }^{R} C(\pi)=-0.603\right.$; for a fully polarized band $\left.{ }^{R} C(\pi)=-1^{[14]}\right)$ and is calculated to occur at

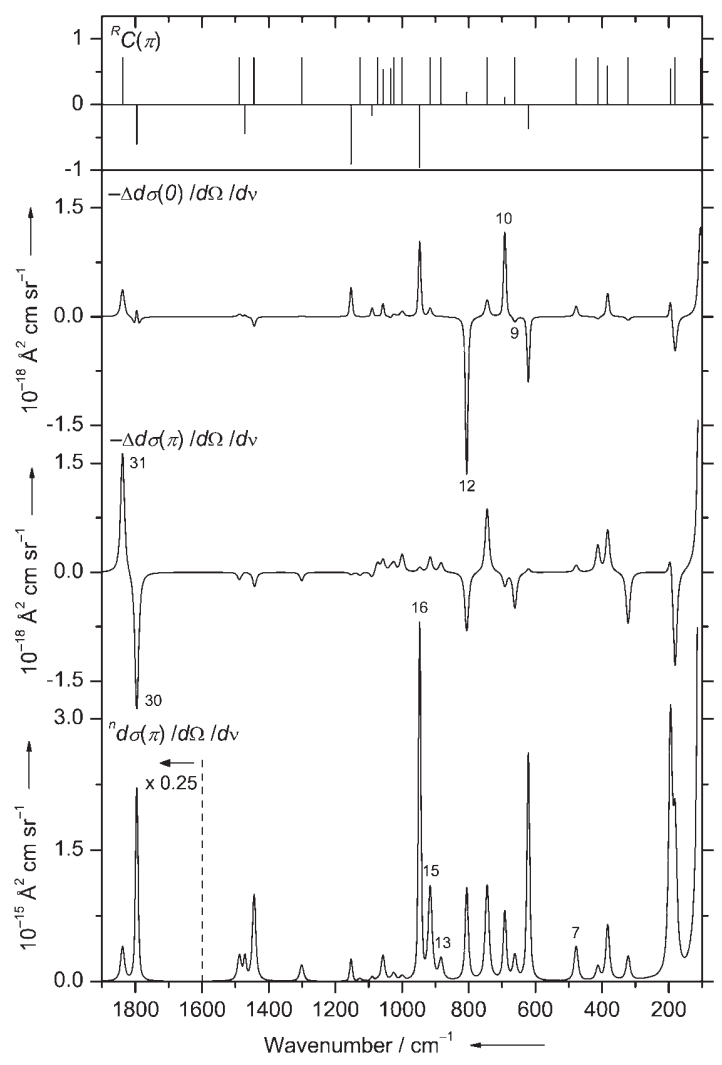

Figure 4. Computed Raman and ROA spectra of $(+)-(P)-1,4-$ dimethylenespiropentane. From bottom to top: Raman backscattering, ROA backscattering, ROA forward scattering, degree of circularity in backscattering. Computational parameters: vibrational modes from density functional theory with B3LYP/aug-cc-pVTZ (Gaussian03); ${ }^{[23]}$ electronic tensors from time-dependent Hartree-Fock with aug-cc-pVDZ (DALTON). ${ }^{[24]}$ Isotropic and anisotropic bandwidths 3.5 and $10 \mathrm{~cm}^{-1}$, respectively. 
$1796 \mathrm{~cm}^{-1}$ with a very high Raman intensity and strongly negative ROA. The much weaker, fully depolarized out-of-phase component (symmetry species $B$, vibration $31,{ }^{R} C(\pi)={ }^{5} / 7$ ) should have equally strong positive ROA and is calculated to occur at $1839 \mathrm{~cm}^{-1}$. The two vibrations are shown in Figure 5 . A detailed discussion of the way in which we represent vibrations is given elsewhere. ${ }^{[15]}$
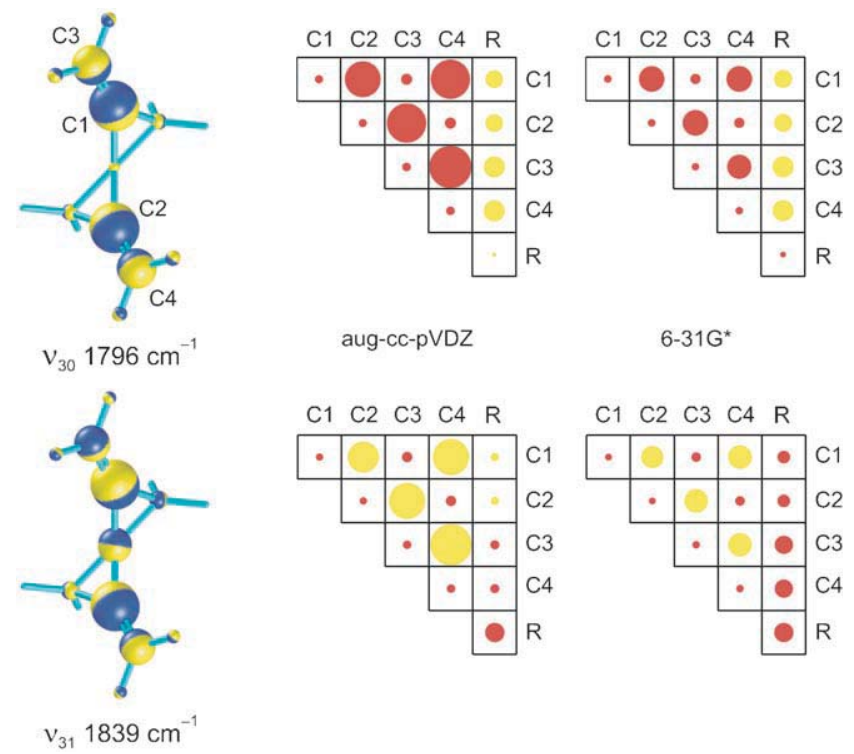

aug-cc-pVDZ

$6-31 G^{*}$
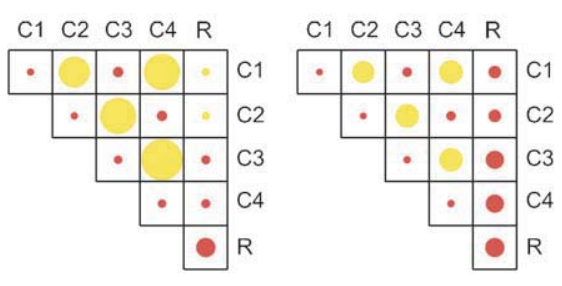

igure 5. The A-symmetric (vibration 30, top) and B-symmetric (vibration 31 , bottom) coupled $C=C$ stretching vibrations with their group-coupling matrices. The volume of the spheres centered on the nuclei is proportional to the vibrational energy of the nuclei, and the direction of their motion is perpendicular to the planes which separate the half-spheres represented in different colors. The five groups in the group-coupling matrices are the four carbon atoms, as indicated, and the fifth group is the remainder of the molecule. Computational parameters: vibrations from density functional theory with B3LYP/aug-cc-pVTZ (Gaussian03); electronic tensors from time-dependent Hartree-Fock with basis sets as indicated (DALTON).

In the experimental ROA spectrum, in contrast, two polarized, fairly intense Raman bands are found at 1710 and $1757 \mathrm{~cm}^{-1}$. The band at $1710 \mathrm{~cm}^{-1}$ is narrow [full-width-at-halfmaximum (FWHM) $9 \mathrm{~cm}^{-1}$; instrumental resolution $7 \mathrm{~cm}^{-1}$ ] and has slightly negative ROA, while the band at $1757 \mathrm{~cm}^{-1}$ is much wider $\left(F W H M=15 \mathrm{~cm}^{-1}\right)$ and associated with a small ROA couplet, decentered with respect to the peak of the Raman band. A far weaker, depolarized Raman band with small positive ROA occurs at $1812 \mathrm{~cm}^{-1}$ with a shoulder at $1800 \mathrm{~cm}^{-1}$.

The two strong, polarized bands can only be explained by Fermi resonance. Both bands must draw their intensity from the A-symmetric component of the coupled $\mathrm{C}=\mathrm{C}$ stretching motions. The large width and the fact that a small couplet rather than a single ROA band is associated with it are indicative that the Raman band at $1757 \mathrm{~cm}^{-1}$ is again made up of two components. Thus, it appears that the vibrational state due to the $\mathrm{C}=\mathrm{C}$ stretching motion of species $\mathrm{A}$ interacts with a minimum of two vibrational states arising from combination and/or overtone states of lower-energy vibrations.
The weak, depolarized Raman band at $1812 \mathrm{~cm}^{-1}$ most likely represents the $\mathrm{B}$-symmetric component of the coupled $\mathrm{C}=\mathrm{C}$ stretching motion, again with the shoulder at $1800 \mathrm{~cm}^{-1}$ possibly owing to Fermi resonance with an overtone or combination state. It follows from comparison of the frequencies computed in the harmonic approximation with the average observed frequencies that anharmonicity lowers the energy of the A-symmetric vibrational state more than that of the B-symmetric one.

The coupling of several vibrational modes was considered by Fermi in his original work, ${ }^{[16]}$ namely, the interaction of the symmetric $\mathrm{CO}_{2}$ stretching vibration estimated at $1230 \mathrm{~cm}^{-1}$ with three vibrational states, generated by overtones of the doubly degenerate bending mode at $673 \mathrm{~cm}^{-1}$. In the $D_{\infty h^{-}}$ symmetric $\mathrm{CO}_{2}$ molecule, only two of these states actually interact with the stretching fundamental, and only two of the three resulting states are observed in the Raman spectrum. The selection rules for the point-group symmetry $C_{2}$ of $(+)-(P)-$ 1,4-dimethylenespiropentane are less restrictive, and the vibrational structure of the molecule is far more complex than that of $\mathrm{CO}_{2}$. It is thus easily conceivable for two A-symmetric overtone or combination frequencies to have the appropriate energy for them to interact with the A-symmetric component of the $\mathrm{C}=\mathrm{C}$ stretching mode. Candidates for overtone frequencies will be considered in the discussion of the solution spectra.

Fermi resonance does not, however, explain why the computed and measured ROA intensities are unrelated. Total ROA intensity should be conserved by Fermi resonance (similar to Raman intensity), and Fermi resonance cannot mix states of different symmetries and thereby lead to an annihilation of the intensities of the states of species A and B. One possible explanation for the intensity discrepancies might be our finding that the computed size of the ROA couplet is strongly dependent on the detailed description of the electron distribution, even when the vibrational motion is kept unchanged. Without the inclusion of valence-orbital type diffuse functions into the basis set, the size of the couplet collapses by an order of magnitude. The group-coupling matrices ${ }^{[15]}$ (Figure 5) for two different basis sets demonstrate that the coupling terms between the carbon atoms of the two double bonds strongly depend on the presence or absence of such diffuse functions. It is thus conceivable that the small size of the ROA measured in the liquid phase is a consequence of the perturbation of the electron distribution by intermolecular interactions.

\section{Comparison of Computed and Measured Data in the 860- $960 \mathrm{~cm}^{-1}$ Region.}

Salient discrepancies also occur between measured and computed Raman data in the $900-950 \mathrm{~cm}^{-1}$ region. The discrepancies persist even if the usual offset between harmonic and anharmonic frequencies is disregarded. According to the calculations, there should be an intense, polarized band at $947 \mathrm{~cm}^{-1}$ (vibration 16 , species $A,{ }^{R} C(\pi)=-0.953$ ) and a less intense, depolarized band at $915 \mathrm{~cm}^{-1}$ arising from two almost degenerate vibrations $\left(15\right.$, species $A,{ }^{R} C(\pi)=0.712$, and 14 , species $B$, 
$\left.{ }^{R} C(\pi)={ }^{5} / 7 \cong 0.714\right)$. In contrast, the experimental spectrum consists of two partially resolved, polarized bands, which peak at 936 and $924 \mathrm{~cm}^{-1}$, with no further Raman band down to $900 \mathrm{~cm}^{-1}$.

Vibration 16, calculated at $947 \mathrm{~cm}^{-1}$, is the in-phase coupling (species A) of the two symmetric half-ring stretching motions ${ }^{[17]}$ involving the $\mathrm{CH}_{2}$ groups of the cyclopropane moieties of the molecule (Figure 6). The two vibrations calculated at 916 and $915 \mathrm{~cm}^{-1}$ represent the in-phase (species $A$, vibration 15) and out-of-phase (species $B$, vibration 14) couplings, respectively, of the localized wagging-type motions of the two

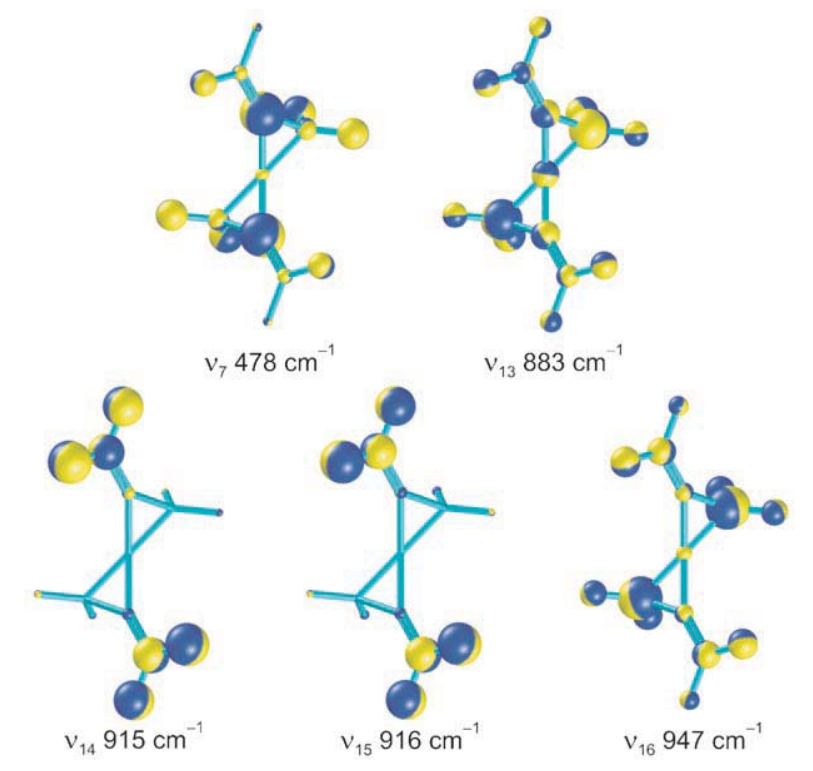

Figure 6. Vibrational energy representation of vibrations $7,13,14,15$, and 16. The volume of the spheres centered on the nuclei is proportional to the vibrational energy of the nuclei, and the direction of their motion is perpendicular to the planes which separate the half-spheres represented in different colors.

$\mathrm{C}=\mathrm{CH}_{2}$ groups. To explain the two polarized peaks observed at 936 and $924 \mathrm{~cm}^{-1}$ without invoking Fermi resonance with an overtone, a very substantial error in the computed position of the symmetric $\mathrm{C}=\mathrm{CH}_{2}$ wagging motion must be assumed in addition to an expected shift towards lower energy of the halfring stretching mode 16 through anharmonicity. If the wagging vibration of species A would actually lie within a few wavenumbers of the in-phase half-ring stretching mode before interacting with it, then anharmonicity might mix and energetically separate these two A-symmetric states, with a concomitant transfer of intensity from the half-ring stretch to the wagging mode. In view of the strongly localized nature of the wagging mode, this is an unlikely explanation for the two intense bands observed at 936 and $924 \mathrm{~cm}^{-1}$.

Fermi resonance of the in-phase half-ring stretching mode 16 with a vibrational state resulting from a combination or overtone frequency appears a more likely cause of the bands peaking at 936 and $924 \mathrm{~cm}^{-1}$. The first overtone of mode 7 , with the fundamental calculated at $478 \mathrm{~cm}^{-1}$ and observed at $465.3 \mathrm{~cm}^{-1}$, appears a good candidate. This A-symmetric vibra- tion is a torsional motion of the planes of the two rings with respect to each other (Figure 6), which should be able to interact strongly with the half-ring stretching motion. We will see below that the solution spectra lend support to such an interpretation.

From the experimental ROA backscattering spectrum in Figure 2, there is evidence that at least one wagging motion occurs, as would be expected, slightly shifted towards lower energy with respect to the computed harmonic frequency of $915 \mathrm{~cm}^{-1}$, with the ROA band peaking at $877 \mathrm{~cm}^{-1}$ and having a distinct shoulder at $890 \mathrm{~cm}^{-1}$. It is tempting to attribute this shoulder to the two wagging modes 14 and 15 and the peak at $877 \mathrm{~cm}^{-1}$ to vibration 13 . The gas-phase Raman and ROA intensities computed for the A-symmetric wagging mode 15 are too high, however, for this assignment to be considered certain. Vibration 13 is an out-of-phase combination of two symmetric half-ring stretch motions of the cyclopropane moieties, as shown in Figure 6.

There is evidence from spectral changes that occur when $(+)-(P)-1,4$-dimethylenespiropentane is dissolved in $\left[D_{3}\right]$ acetonitrile that the $A$-symmetric wagging mode 15 might actually be hidden under the $877 \mathrm{~cm}^{-1}$ peak in Figure 2, as the solution spectrum displays a substantial shoulder also visible in the Raman spectrum at $890 \mathrm{~cm}^{-1}$. The pertinent data are included in the section on solvent influence but they are not further discussed. We feel that without explicitly taking into account the effects of the condensed phase and of anharmonicity in a computation, it is not possible to uniquely identify the three vibrations 13,14 , and 15 in the experimental spectra.

\section{Spectra in Solution and Forward Scattering Spectra}

\section{Solvent Choice}

A major problem in measuring Raman and ROA solution spectra is obscuration of solute by solvent bands. A further problem, of a technical nature and unique to optical multichannel detection, is saturation of the CCD detector by sharp, intense solvent bands; this condition tends to lead to offsets in ROA spectra. Small polar molecules such as $\mathrm{H}_{2} \mathrm{O}$ produce little Raman scattering and are thus suitable solvents, but they are not usable for a compound like (+)-(P)-1,4-dimethylenespiropentane.

We have found acetonitrile and $\left[\mathrm{D}_{3}\right]$ acetonitrile to often be good choices in such situations. They have few Raman bands in the 200 to $2000 \mathrm{~cm}^{-1}$ range, none of which are excessively strong. Despite its large dipole moment of $3.92 \mathrm{D}$ and the substantial dielectric constant of 38.8, acetonitrile is able to dissolve many unsaturated hydrocarbons. (+)-(P)-1,4-dimethylenespiropentane proved miscible. The combined use of the deuterated and non-deuterated form also provides an uninterrupted spectral coverage throughout the $200-2000 \mathrm{~cm}^{-1}$ range.

However, only solutions in $\left[D_{3}\right]$ acetonitrile were measured, because a total of $63 \mathrm{mg}$ of $(+)-(P)-1,4$-dimethylenespiropentane was available for all measurements, and this volatile and 
unstable compound cannot easily be recovered from a solvent like acetonitrile. $\left[\mathrm{D}_{3}\right]$ acetonitrile was preferred, because it shows little Raman scattering in the $880-980 \mathrm{~cm}^{-1}$ region and none in the $1500-2000 \mathrm{~cm}^{-1}$ spectral range in which the most prominent discrepancies between computed and measured spectra occur.

The concentration was chosen as $20 \mathrm{vol} \%$. Assuming that the unknown density of $(+)-(P)-1,4$-dimethylenespiropentane is similar to the density of $\left[D_{3}\right]$ acetonitrile, that is, $0.84 \mathrm{~g} \mathrm{~mL}^{-1}$, $20 \mathrm{vol} \%$ corresponds to a molar fraction of 0.107 . Thus, there will be between eight and nine solvent molecules for each solute molecule.

Figure 7 shows the backscattering Raman spectrum of $\left[\mathrm{D}_{3}\right]$ acetonitrile as a hatched area superimposed on the Raman spectrum of the $20 \mathrm{vol} \%$ solution of $(+)-(P)-1,4$-dimethylenespiropentane. The scattering intensities are normalized so that the heights of the two largest Raman peaks of $\left[\mathrm{D}_{3}\right]$ acetonitrile in the $100-1900 \mathrm{~cm}^{-1}$ spectral range are the same for the neat $\left[\mathrm{D}_{3}\right]$ acetonitrile and the solution. The solution backscattering

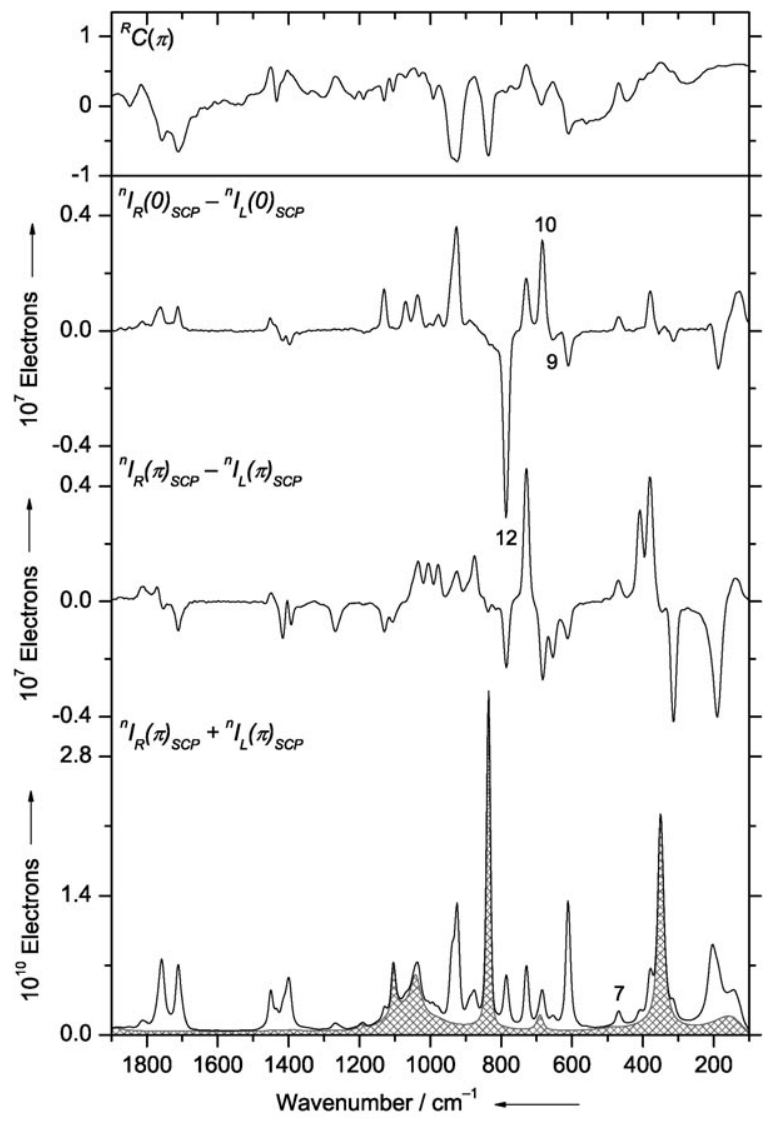

Figure 7. Experimental Raman and ROA spectra of $(+)-(P)-1,4$-dimethylenespiropentane measured as 20 vol\% solutions in $\left[D_{3}\right]$ acetonitrile on the Fribourg ROA instrument. From bottom to top: Raman backscattering, ROA backscattering, ROA forward scattering, degree of circularity for backscattering. Raman forward scattering is identical to backscattering except for a reduced throughput of the fiber optics used in the instrument; ROA forward scattering has therefore been adjusted so as to correspond to the same throughput as backscattering. The hatched area corresponds to the Raman spectrum of $\left[\mathrm{D}_{3}\right]$ acetonitrile. Exposure time $90 \mathrm{~min}$, laser power at sample $150 \mathrm{~mW}$, exciting wavelength $532 \mathrm{~nm}$, resolution $7 \mathrm{~cm}^{-1}$, average spectral width of one column of the CCD detector $2.4 \mathrm{~cm}^{-1}$, sample size $28 \mu \mathrm{L}$. and forward scattering ROA spectra of $(+)-(P)-1,4$-dimethylenespiropentane, included in Figure 7 , show a remarkable absence of offset for the spectral positions of the strong solvent bands.

\section{Influence of the Solvent Environment on Fermi Resonances in the $\mathrm{C}=\mathrm{C}$ Stretching Region}

The most prominent change in the 1700 to $1790 \mathrm{~cm}^{-1} \mathrm{C}=\mathrm{C}$ inphase stretching region of the Raman spectrum of $(+)-(P)-1,4-$ dimethylenespiropentane upon dissolving the compound in $\left[\mathrm{D}_{3}\right]$ acetonitrile is a narrowing and an increase in height of the intense broad band at $1757 \mathrm{~cm}^{-1}$ (Figure 8). The separation and relative size of the positive and negative peaks of the ROA backscattering couplet associated with this Raman band change little in solution. It therefore appears that it is less the separation of the two vibrational states, that is responsible for the associated wide Raman band which changes, but the size of their individual Raman intensities. A shift of intensity from the higher frequency component to the lower frequency one,

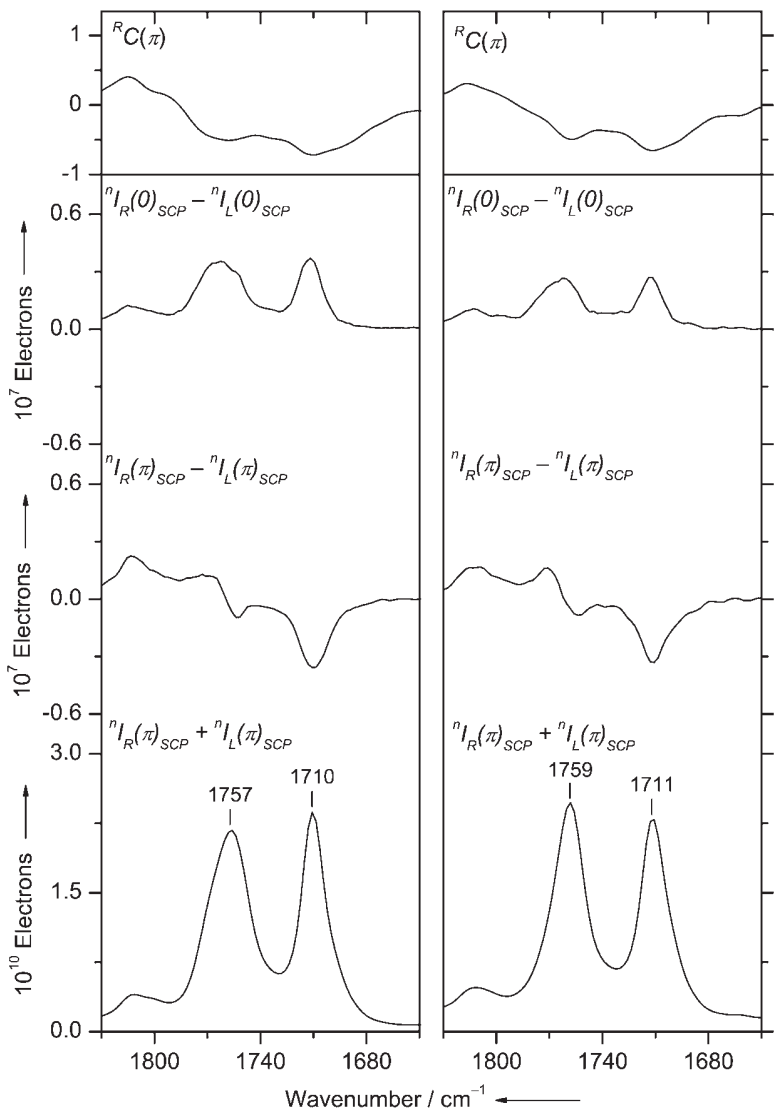

Figure 8. Comparison of the Raman and ROA bands of (+)-(P)-1,4-dimethylenespiropentane measured as a neat liquid (left) and as a 20 vol\% solution in $\left[D_{3}\right]$ acetonitrile (right) for the 1650 to $1830 \mathrm{~cm}^{-1}$ region. The arrangement from bottom to top is as in Figures 2 and 7. Forward scattering ROA intensities are adjusted relative to backscattering for reduced instrumental throughput. The relative scattering intensities of the neat liquid and the $\left[D_{3}\right]$ acetonitrile solution were normalized so that the largest peaks in the two measured spectra (in both cases vibration 8 at $609 \mathrm{~cm}^{-1}$ ) have the same height. 
along with a slight displacement towards higher energy, would explain the observed changes.

The separation of $46 \mathrm{~cm}^{-1}$ between the two Raman bands at 1757 and $1710 \mathrm{~cm}^{-1}$, which are both drawing their intensity from the in-phase combination of the stretching motion of the two $\mathrm{C}=\mathrm{C}$ bonds, does not change noticeably in solution, though both bands shift by 1 to $2 \mathrm{~cm}^{-1}$ towards higher wavenumbers.

The question as to which fundamentals those states belong which interact with the in-phase $\mathrm{C}=\mathrm{C}$ stretching vibration cannot be answered with certainty. If we first look only at overtones, then, from the point of view of symmetry, fundamentals of both species, A and B, must be considered. The requirement that the energy of the fundamentals in question should be slightly higher than approximately half the energy of the noninteracting $\mathrm{C}=\mathrm{C}$ stretching fundamental, which must be in the vicinity of $1740 \mathrm{~cm}^{-1}$, narrows the choice to fundamentals with a frequency in the $870-890 \mathrm{~cm}^{-1}$ range. Unfortunately, this region is one where we have found it difficult to uniquely identify vibrational states. A likely candidate is certainly the first overtone of vibration 13, the out-of-phase combination of the symmetric half-ring stretch motions of the cyclopropane moieties, but the wagging vibrations 14 and 15 discussed earlier are also candidates. Combination states of different fundamentals are another possibility.

It is important to note that the wagging motions are localized on the light hydrogen nuclei of the two $\mathrm{C}=\mathrm{CH}_{2}$ fragments. This means that the excursion of these nuclei is large, and vibrations with this property tend to be anharmonic. The frequency of the first overtone can then be substantially lower than twice the value of the fundamental. The involvement of a vibration with a large amplitude would help to explain the strong dependence on intermolecular interactions found for the shape of the $1757 \mathrm{~cm}^{-1}$ band.

\section{Influence of the Solvent Environment in the 860 to $960 \mathrm{~cm}^{-1}$ Region}

The most interesting change in the Raman spectrum occurs for the double-peaked band centered at $930 \mathrm{~cm}^{-1}$ and attributed in the preceding section to Fermi resonance. Instead of a main maximum at $936 \mathrm{~cm}^{-1}$ and a secondary one at $924 \mathrm{~cm}^{-1}$, the solution Raman spectrum in $\left[\mathrm{D}_{3}\right]$ acetonitrile has a maximum at $924 \mathrm{~cm}^{-1}$ and a mere shoulder at about $938 \mathrm{~cm}^{-1}$. The spectra of the compound as a neat liquid and in $\left[\mathrm{D}_{3}\right]$ acetonitrile solution are compared in Figure 9 on an expanded scale.

The changes in the Raman intensities suggest that the energetic positions of the two vibrational states in Fermi resonance are interchanged when (+)-( $(P)$-1,4-dimethylenespiropentane is dissolved in $\left[D_{3}\right]$ acetonitrile. The fact that the degree of circularity is always slightly more negative for the vibrational state with the larger Raman intensity, independent of whether it is the high or low energy one, also points in this direction.

The energy of the fundamental 7, the overtone of which we consider the likely candidate for interacting with the fundamental of mode 16 , depends in the required way on the solvent environment. In $\left[\mathrm{D}_{3}\right]$ acetonitrile, the band of the funda-

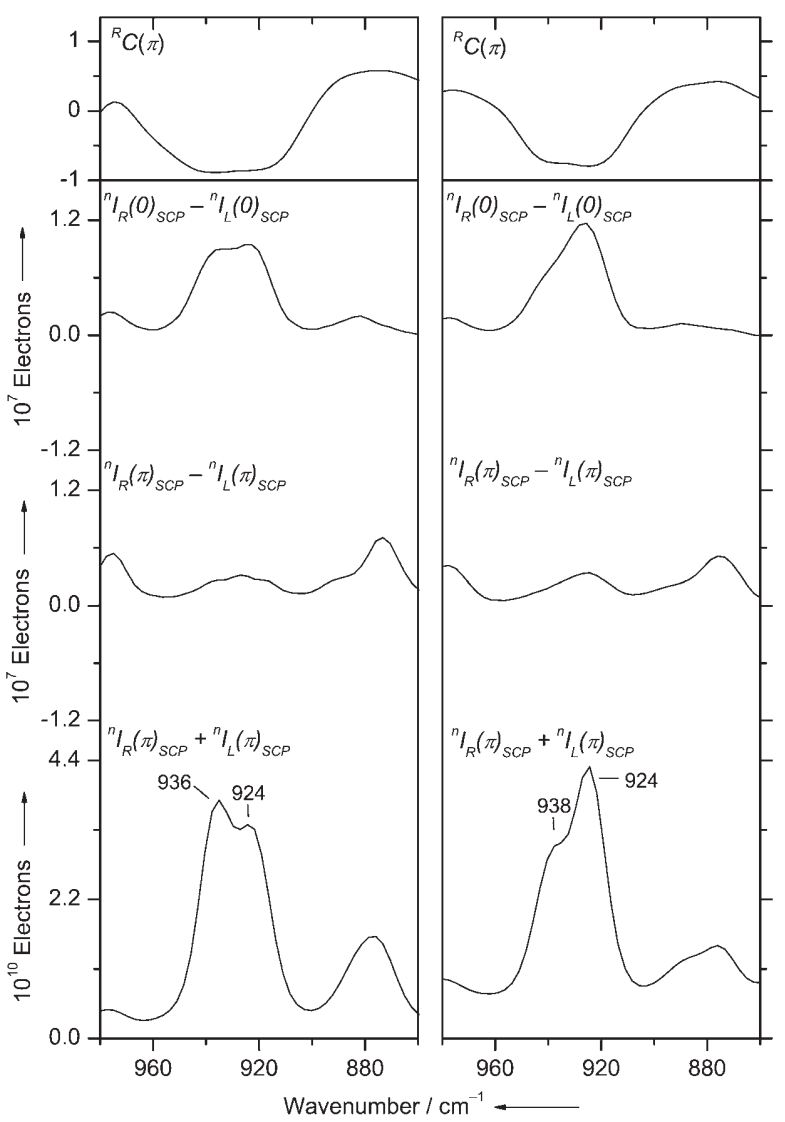

Figure 9. Comparison of the Raman and ROA bands of (+)-(P)-1,4-dimethylenespiropentane measured as a neat liquid (left) and as a 20 vol\% solution in $\left[D_{3}\right.$ ]acetonitrile (right) for the 860 to $980 \mathrm{~cm}^{-1}$ region. The arrangement from bottom to top is as in Figures 2 and 7. Forward scattering ROA intensities are adjusted relative to backscattering for reduced instrumental throughput. The relative scattering intensities of the neat liquid and the $\left[\mathrm{D}_{3}\right]$ acetonitrile solution were normalized so that the largest peaks in the two measured spectra (in both cases vibration 8 at $609 \mathrm{~cm}^{-1}$ ) have the same height.

mental 7 peaks at $468.2 \mathrm{~cm}^{-1}$, which is almost $3 \mathrm{~cm}^{-1}$ higher than what we observe in the neat liquid. The first overtone is then expected in the vicinity of, or slightly below, the energy corresponding to twice the frequency of the fundamental, that is, $930.6 \mathrm{~cm}^{-1}$ for the neat liquid and $936.4 \mathrm{~cm}^{-1}$ in $\left[\mathrm{D}_{3}\right]$ acetonitrile. The fundamental of mode 16 , before interacting with the overtone, has to fall between these two limits, corrected for a possible stronger influence of anharmonicity on the overtone than on the fundamental of mode 7 . The required value of approximately $930 \mathrm{~cm}^{-1}$ is compatible with the value of $947 \mathrm{~cm}^{-1}$ computed in the harmonic approximation.

In order to obtain a better estimate of the separation of the Fermi resonance states than is possible by visual inspection of the measured curves, we applied curve-resolution techniques based on simple Lorentzian functions. For the Raman spectrum of the neat liquid, we found values of 936 and $921.8 \mathrm{~cm}^{-1}$ for the underlying vibrational states, while in $\left[D_{3}\right]$ acetonitrile solution, the corresponding values are 939.1 and $924.3 \mathrm{~cm}^{-1}$. The separation of 14 to $15 \mathrm{~cm}^{-1}$ is similar to what is estimated from the ROA spectra of the neat liquid (in backward and for- 
ward scattering), while in $\left[D_{3}\right]$ acetonitrile solution the separation appears slightly larger, on the order of $18 \mathrm{~cm}^{-1}$ for both scattering geometries.

A remarkable change in the forward ROA scattering spectrum in going from neat substance to $\left[D_{3}\right]$ acetonitrile solution is the increase in intensity of the low-frequency component of the Fermi-resonance doublet. A similar increase is absent in backward scattering.

\section{Influence of the Solvent Environment in Other Spectral Regions}

The general tendency upon dissolution of $(+)-(P)-1,4$-dimethylenespiropentane in $\left[D_{3}\right]$ acetonitrile is a shift of most Raman and ROA bands by a few wavenumbers towards higher values. From standard theories on the influence of the condensed phase on Raman scattering, ${ }^{[18-20]}$ one also expects a reduction per molecule of the scattering intensities because of the almost certainly lower index of refraction of 1.344 (sodium D-line) of $\left[D_{3}\right]$ acetonitrile compared to the unknown one of $(+)-(P)-1,4-$ dimethylenespiropentane. If the value of the index of refraction of 1.496 (sodium $D$-line) of the isomeric toluene were to hold for $(+)-(P)-1,4$-dimethylenespiropentane, then the calculated reduction for a $20 \mathrm{vol} \%$ solution should be 30 to $40 \%$.

We do observe a reduction in the Raman scattering intensity, but the effective light-collection angle of the non-imaging light-collection optics ${ }^{[10]}$ and the length of the segment of the focused laser beam from which light is collected, vary in our instrument with the refraction index of the sample. A quantitative comparison of the variation of the experimental and theoretical intensities as a function of the refraction index is therefore not meaningful.

There is a notable absence of any striking change in the shape of either the Raman or the ROA signals in the 1350 to $1500 \mathrm{~cm}^{-1}$ region upon dissolving (+)-(P)-1,4-dimethylenespiropentane in trideuterioacetonitrile, even though Fermi resonance almost certainly plays a role in this spectral region. We conclude this from the lack of detailed agreement between the computed and measured spectra. The Raman intensity in this region is due to the four coupled scissoring motions of the molecule, and it is not obvious which of the four fundamentals may interact with which of the many overtone and/or combination states whose energy is in the 1350 to $1500 \mathrm{~cm}^{-1}$ range.

\section{Conclusions}

1,4-Dimethylenespiropentane does not conform to the notion that small, nonpolar molecules without internal degrees of freedom are easy examples for a reliable ab initio computation of vibrational optical activity. Its experimental Raman and ROA spectra show sizable bands that have no counterparts in the calculated spectra, and the two most prominent computed ROA signals, the components of a large couplet due to $C=C$ stretching motions, are not present in the measured spectrum.
The virtual absence of the computed couplet in the experimental ROA backscattering spectrum appears to be due to intermolecular interactions in the condensed phase. We reach this conclusion from observing an extreme dependence of the size of this couplet on the detailed computational description of the electron distribution of 1,4-dimethylenespiropentane, and in particular on the inclusion or omission of diffuse valence-type functions in the basis set.

The presence of large Raman and ROA signals in the 920 to $940 \mathrm{~cm}^{-1}$ and 1700 to $1800 \mathrm{~cm}^{-1}$ regions, which cannot be accounted for by a computation done within the harmonic approximation, and for which we can exclude impurities as the source, must be attributed to Fermi resonances.

In the 1700 to $1800 \mathrm{~cm}^{-1}$ region, the fundamental of the symmetric combination of the two $\mathrm{C}=\mathrm{C}$ stretching motions, estimated at $1740 \mathrm{~cm}^{-1}$, appears to interact with the first overtone of the out-of-phase symmetric half-ring stretching vibration. Other candidates are the $\mathrm{C}=\mathrm{CH}_{2}$ wagging motions, the fundamentals of which also occur in the $870-900 \mathrm{~cm}^{-1}$ range. The combined evidence from the Raman and ROA spectra measured for the neat liquid and for its solutions point to multiple Fermi resonances.

In the $920-940 \mathrm{~cm}^{-1}$ region, the fundamental of the symmetric combination of the half-ring stretching motions involving the $\mathrm{CH}_{2}$ groups of the two cyclopropane rings, estimated at $930 \mathrm{~cm}^{-1}$, interacts with the first overtone of the twisting motion of the two rings relative to each other, the fundamental of which occurs at $465.3 \mathrm{~cm}^{-1}$. This conclusion is supported by the strong solvent dependence of the Fermi resonance bands.

We have characterized the fundamental at $1740 \mathrm{~cm}^{-1}$ as a combination of two $\mathrm{C}=\mathrm{C}$ stretching motions. Looking at the displacements of individual nuclei, one could equally well call it a symmetric combination of two half-ring stretching motions involving the quaternary $\mathrm{C}$-atoms of the $\mathrm{C}=\mathrm{C}$ bonds. This second designation makes it clear that a common aspect of the $930 \mathrm{~cm}^{-1}$ and the $1740 \mathrm{~cm}^{-1}$ fundamental vibration is the symmetric stretching of a central triatomic C-C-C entity of 1,4dimethylenespiropentane. Such stretching motions appear prone to interact with overtone and combination states, and Fermi himself, in an addendum to his paper on $\mathrm{CO}_{2}{ }^{[16]}$ pointed out the symmetric stretching vibrations of $\mathrm{CS}_{2}$ and $\mathrm{CCl}_{4}$ as further examples of vibrations leading to resonance.

How important is Fermi resonance in [4]triangulane, the cyclopropane analogue of 1,4-dimethylenespiropentane? In earlier work, ${ }^{[1]}$ we found excellent agreement between the measured and computed Raman and ROA data. Recent, more elaborate computations have led to the same result. ${ }^{[5]}$ Fermi resonance interaction undoubtedly exists in this vibrationally complex molecule but does not show up prominently in either its Raman or ROA spectrum. It is an old, but not generally wellknown spectroscopic truth, ${ }^{[21]}$ though, that Fermi resonances tend to be far more visible in vibrational absorption than in Raman scattering spectra. This difference might be at the root of a lack of agreement between computed and measured VCD spectra found for the triangulanes and for 1,4-dimethylenespiropentane. ${ }^{[22]}$ The disagreement was attributed to the presence 
of impurities, and the VCD data of these unique molecules were not investigated further.

The lower sensitivity of ROA than of VCD to Fermi resonance is one of the important analytical advantages of ROA measurements. Another one is the possibility to measure the degree of circularity, which can yield information on the nature of vibrational states that does not depend on a computation. In the case of the $C_{2}$-symmetric 1,4-dimethylenespiropentane, any Raman band for which the degree of circularity deviates from $5 / 7$ must be due to a vibrational state of species $A$. The negative values of the measured degree of circularity are, therefore, direct experimental proof of the A-symmetric nature of the Fermi resonances in the 920 to $940 \mathrm{~cm}^{-1}$ and 1700 to $1800 \mathrm{~cm}^{-1}$ regions.

Herein, we have emphasized the rich spectroscopic information which ROA can provide by focusing on discrepancies between observed and computed data more than on agreement. If we take a stereochemist's rather than a spectroscopist's point of view, it is also obvious that the comparison of the computed and the measured data would have allowed the determination of the absolute configuration of the measured enantiomers of 1,4-dimethylenespiropentane if it had not been known. The structural information obtainable through ROA is certainly its practically most important aspect. The present work also demonstrates that ROA, with the recent availability of high-quality experimental data, is a potent spectroscopic tool in its own right.

\section{Acknowledgements}

For the Swiss group, this work was supported by the Swiss $\mathrm{Na}$ tional Foundation for Science (grants 200020-103750/1, 200020112201/1). For the German group, this work was supported by the State of Niedersachsen and the Fonds der Chemischen Industrie. The authors are grateful to Daniel Frank, Göttingen, for the re-preparation of $(+)-(P)-1,4-$ dimethylenespiropentane.
[4] G. Zuber, W. Hug, Helv. Chim. Acta 2004, 87, 2208.

[5] M. Reiher, V. Liégeois, K. Ruud, J. Phys. Chem. A 2005, 109, 7567.

[6] K. J. Jalkanen, R. M. Nieminen, K. Frimand, J. Bohr, H. Bohr, R. C. Wade, E. Tajkhorshid, S. Suhai, Chem. Phys. 2001, 265, 125.

[7] K. J. Jalkanen, R. M. Nieminen, M. Knapp-Mohammady, S. Suhai, Int. J. Quantum Chem. 2003, 92, 239.

[8] A. de Meijere, A. F. Khlebnikov, S. I. Kozhushkov, R. R. Kostikov, P. R. Schreiner, A. Wittkopp, C. Rinderspracher, D. S. Yufit, J. A. K. Howard, Chem. Eur. J. 2002, 8, 828.

[9] W. Hug, S. I. Kozhushkov, K. Rauch, A. de Meijere, unpublished results. [10] W. Hug, G. Hangartner, J. Raman Spectrosc. 1999, 30, 841.

[11] W. Hug, Appl. Spectrosc. 2003, 57, 1.

[12] "Construction of a new forward and backward scattering Raman optical activity spectrometer and graphical analysis of measured and calculated spectra for (R)-[ $\left[\mathrm{H}_{1},{ }^{2} \mathrm{H}_{2},{ }^{2} \mathrm{H}_{3}\right]$-neopentane," J. Haesler, PhD thesis, Université de Fribourg, Switzerland, 2006.

[13] L. D. Barron, Molecular Light Scattering and Optical Activity, Cambridge University Press, Cambridge, 2004

[14] D. A. Long, The Raman Effect, Wiley, Chichester, 2002.

[15] W. Hug, Chem. Phys. 2001, 264, 53.

[16] E. Fermi, Z. Phys. 1931, 71, 250.

[17] N. B. Colthup, L. H. Daly, S. E. Wiberly, Infrared and Raman Spectroscopy, Academic Press, New York, 1975.

[18] G. Eckhart, W. G. Wagner, J. Mol. Spectrosc. 1966, 19, 407.

[19] N. K. Sidorov, L. S. Stalmakhova, N. V. Bogachyov, Opt. Spectrosc. 1971, $30,375$.

[20] H. W. Schrötter, H. W. Klöckner in Raman Spectroscopy of Gases and Liquids (Ed.: A. Weber), Springer, Berlin, 1979, p. 123.

[21] L. A. Woodward in Raman Spectroscopy, Theory and Practice (Ed.: H. A. Szymansky), Plenum, New York, 1970, p. 1.

[22] P. Stephens, unpublished results.

[23] Gaussian 03, Revision C.01, M. J. Frisch, G. W. Trucks, H. B. Schlegel, G. E. Scuseria, M. A. Robb, J. R. Cheeseman, J. A. Montgomery, Jr., T. Vreven, K. N. Kudin, J. C. Burant, J. M. Millam, S. S. lyengar, J. Tomasi, V. Barone, B. Mennucci, M. Cossi, G. Scalmani, N. Rega, G. A. Petersson, H. Nakatsuji, M. Hada, M. Ehara, K. Toyota, R. Fukuda, J. Hasegawa, M. Ishida, T. Nakajima, Y. Honda, O. Kitao, H. Nakai, M. Klene, X. Li, J. E. Knox, H. P. Hratchian, J. B. Cross, V. Bakken, C. Adamo, J. Jaramillo, R. Gomperts, R. E. Stratmann, O. Yazyev, A. J. Austin, R. Cammi, C. Pomelli, J. W. Ochterski, P. Y. Ayala, K. Morokuma, G. A. Voth, P. Salvador, J. J. Dannenberg, V. G. Zakrzewski, S. Dapprich, A. D. Daniels, M. C. Strain, O. Farkas, D. K. Malick, A. D. Rabuck, K. Raghavachari, J. B. Foresman, J. V. Ortiz, Q. Cui, A. G. Baboul, S. Clifford, J. Cioslowski, B. B. Stefanov, G. Liu, A. Liashenko, P. Piskorz, I. Komaromi, R. L. Martin, D. J. Fox, T. Keith, M. A. Al-Laham, C. Y. Peng, A. Nanayakkara, M. Challacombe, P. M. W. Gill, B. Johnson, W. Chen, M. W. Wong, C. Gonzalez, J. A. Pople, Gaussian, Inc., Wallingford, CT, 2004.

[24] Dalton, a molecular electronic structure program, release 1.1, 2000; see: http://www.kjemi.uio.no/software/dalton/dalton.html.

[1] W. Hug, G. Zuber, A. de Meijere, A. Khlebnikov, H.-J. Hansen, Helv. Chim. Acta 2001, 84, 1.

[2] K. Ruud, T. Helgaker, P. Bouř, J. Phys. Chem. A 2002, 106, 7448.

[3] G. Zuber, W. Hug, J. Phys. Chem. A 2004, 108, 2108. 